\title{
El problema del rezago tecnológico de las microempresas industriales y alternativas de solución
}

\author{
Juan Ollivier Fierro*
}

\section{Resumen}

El presente artículo pretende analizar la problemática relacionada con el rezago tecnológico en la industria manufacturera y su relación con variables económicas claves con el fin de proponer alternativas de solución a las microempresas. Para la colección de los datos, se desarrolló un instrumento que se aplicó a una muestra aleatoria estratificada de 125 empresas de la industria manufacturera de la ciudad de Chihuahua, México, en el 2004. Se planteó como hipótesis que el nivel tecnológico del proceso está positivamente relacionado con el tamaño y los resultados económicos de las empresas y se logró identificar el componente tecnológico que ejerce la mayor influencia en el valor agregado a través del análisis de regresión. Con el fin de visualizar las alternativas de solución, se analizó el comportamiento de las empresas mayores (a las micros) para generar un modelo de crecimiento que pondera las relaciones entre las principales variables, agrupadas en factores. Soportadas en este modelo, se propuso como alternativa para incrementar el valor agregado por trabajador en las microempresas un incremento significativo en la inversión para la automatización de los procesos administrativos, acompañado de un mayor impulso a la capacitación del personal.

Palabras clave: tecnología del proceso, microempresa, valor agregado por trabajador, tecnologías en información, tecnologías organizativas.

\section{Introducción}

Dado el cambio en las reglas del juego, impulsado por el vertiginoso desarrollo de las tecnologías e información y comunicaciones (TIC), que entre otras cosas refuerza al fenómeno creciente de la globalización que es entendida como la apertu-

\footnotetext{
* Profesor de la Facultad de Contaduría y Administración de la Universidad Autónoma de Chihuahua.
} 
ra progresiva a los intercambios comerciales, financieros y de información en las economías en el nivel mundial, la situación de las empresas industriales en los países en desarrollo atraviesa por una crisis de falta de competitividad frente al embate de las empresas extranjeras cada vez más presentes en dichos países, con sus consecuencias sobre la economía y el desarrollo local (Berumen, 2006).

La intensidad de esta crisis en las empresas se presenta por lo general en relación inversa con el tamaño de la misma; es decir, las empresas pequeñas son más vulnerables a estos cambios de las reglas del juego debido a que principalmente son empresas con una capacidad limitada orientadas a los mercados locales, donde ahora tienen que competir con las empresas extranjeras. Dicho de otra forma, sufren las desventajas de la globalización sin poder obtener las ventajas de la misma, tal como lo describió gráficamente el ministro de Corea del Sur, Mr. Kim, en la reunión de los países de Asia Pacífico, APEC, realizada en el 2005:

Globalización significa que una pequeña empresa localizada en una parte del Mundo puede ser adversamente influenciada por factores del otro lado del mundo.

Esta situación de desventaja de la pequeña empresa manufacturera, particularmente la micro (hasta 10 trabajadores), es la que motivó el presente trabajo con el ánimo de analizar el problema desde un punto de vista objetivo para proponer alternativas de solución al mismo.

La importancia del problema es relativamente conocida. De acuerdo con el Instituto Nacional de Estadística, Geografía e Informática (INEGI, 2004), se estima que de los 328,718 establecimientos de la industria manufacturera que en el 2003 había en México, y que además generaron aproximadamente 49\% del Producto Bruto del país, el 91\% fueron microempresas que emplearon cerca de 762,103 trabajadores. Estas cifras oficiales, que dimensionan la importancia social del problema y de su potencial beneficio a la comunidad, se quedan cortas cuando se consideran las microempresas no registradas, tal como lo reporta Clemente Ruiz D. (2006); en México se tiene un sector creciente de la economía informal que hoy emplea a más de una tercera parte de la población, además de que las empresas creadas en este sector son de muy bajo nivel agregado, lo cual ha generado un círculo de pobreza.

Sin embargo, afortunadamente, no todo son desventajas; en un análisis rápido de las empresas pequeñas se observan como las principales fortalezas la flexibilidad 
—que incluye capacidad para adaptarse a nuevas situaciones-, la cercanía en el trato con los clientes, la rapidez en la toma de decisiones y la orientación hacia el cliente (Hansen, 2002). En contrapartida, sus principales debilidades son carencia de medios de financiamiento, bajo nivel educativo y recursos de entrenamiento, dependencia de las redes existentes y falta de visión, así como capacidad para innovar.

Cuando se habla de capacidad de una empresa se puede distinguir un aspecto cuantitativo y uno cualitativo. El primero se refiere al volumen máximo de producción que puede alcanzar una unidad productiva en un periodo, que determina dentro del concepto de las denominadas economías de escala, el "tamaño mínimo eficiente" (Mansfield, 1993) que corresponde a la cantidad mínima de producción que debe tener capacidad una empresa para poder ser competitiva en un mercado. Con esta perspectiva, las empresas pequeñas se encuentran en franca desventaja y prácticamente impotentes de competir frente a las grandes empresas, debido a que generalmente se encuentran por debajo del umbral de este "tamaño mínimo eficiente", el cual a su vez, es determinado por razones tecnológicas.

Sin embargo, el aspecto cualitativo, que se refiere más bien a las características de los productos, es un terreno en el que pueden competir con menos desventaja las empresas pequeñas frente a las grandes, pues adecuan sus productos a los nichos de los mercado locales y de esta forma obtienen provecho de su mayor ventaja: su flexibilidad. Esta capacidad de la empresa, relacionada con las características de los productos, se encuentra estrechamente ligada a la manera de cómo se producen los productos, es decir, en la tecnología empleada en los procesos de producción.

Existe la creencia general de que el desarrollo tecnológico requiere de grandes equipos, laboratorios y científicos (Gaynor, 1996), lo cual puede ser cierto en el caso de las grandes empresas, pero éstas sólo representan una fracción de los establecimientos industriales (Conacyt, 2004). La observación más detenida demuestra que la tecnología, en su sentido amplio, está presente en alguna medida en cualquier empresa por pequeña que ésta sea. En consecuencia, se tiene un amplio espectro en el nivel o grado de incorporación de tecnología en los diferentes establecimientos industriales, el cual puede ir desde un mínimo — con el empleo de métodos rudimentarios - hasta un máximo - con una tecnología de punta-, tanto en los procesos como en los productos de la industria (Ollivier, 2005). 
El tema de la presente investigación se encuentra relacionado con el nivel tecnológico del proceso empleado en la producción en las pequeñas empresas manufactureras en los países en desarrollo (Thirlwall, 2003); así, se tomó como caso de estudio las empresas de la industria manufacturera de la ciudad de Chihuahua, México, cuyo estrato más pequeño, que va de uno a diez trabajadores, son las denominadas microempresas. El problema general que se observa es la falta de información sobre este nivel tecnológico del proceso empleado y sus efectos económicos en las microempresas manufactureras, así como las posibles alternativas para mejorarlo en su caso. De este problema general se desprenden las siguientes interrogantes:

1. ¿Cuáles son las principales características de la tecnología empleada en los procesos de las empresas de la industria manufacturera?

2. ¿Cuáles son las relaciones entre los principales factores que inciden en la tecnología de los procesos y sus efectos económicos en las empresas de la industria manufacturera?

3. ¿Cuáles son las alternativas de solución para incrementar el nivel tecnológico y sus efectos económicos en las empresas de la industria manufacturera, particularmente de las micro?

De la problemática anterior resulta un objetivo general que se orienta a analizar el problema del nivel tecnológico del proceso y su relación con los resultados económicos en las empresas de la industria manufacturera, particularmente de las micro, para identificar las alternativas de solución. De él se desprenden los siguientes objetivos específicos:

1. Desarrollar un instrumento que permita conocer las principales características de la tecnología empleada en los procesos y sus efectos económicos en las empresas de la industria manufacturera.

2. Analizar las relaciones entre las principales variables involucradas en el nivel de la tecnología de los procesos y los resultados económicos de las empresas de la industria manufacturera.

3. Identificar las alternativas de solución para incrementar el nivel tecnológico del proceso y el valor agregado en las empresas de la industria manufacturera, particularmente de las micro.

El hecho de medir el nivel tecnológico del proceso en las empresas de la industria manufacturera — particularmente las micro—y relacionarlo con sus resultados 
económicos, así como con las alternativas para mejorarlo, ofrece beneficios potenciales múltiples en la esfera gubernamental, empresarial o académica, los cuales aportarían elementos importantes para el diseño de políticas públicas y programas diferenciados de apoyo a las microempresas, orientados a su desarrollo tecnológico para lograr su sobrevivencia, crecimiento e impacto sobre el desarrollo local, por la vía del incremento de su competitividad, que puede verse a través de la interacción entre tres factores: incremento a la productividad, incremento a la inversión y el empleo (Ruiz, 2004).

\section{Método}

Se trata de un estudio eminentemente empírico, bajo un enfoque cuantitativo, de nivel correlacional, basado en un estudio de campo que tuvo como fuente de información principal una encuesta a una muestra representativa de las empresas de la industria manufacturera en una ciudad del norte de México. La muestra fue del tipo aleatorio estratificado, tomando como estratos los cuatro tamaños de empresas del sector manufacturero. De esta forma se logró dividir la población en dos subgrupos: el estrato de las microempresas y el estrato de las empresas mayores (como se denominan en lo subsecuente), que agrupa a las pequeñas, medianas y grandes, lo cual permitió aislar, para analizar separadamente, el comportamiento de los dos subgrupos.

La primera parte del análisis de nivel descriptivo consistió en identificar y contrastar las principales características económicas y tecnológicas entre los dos subgrupos de empresas, lo cual permitió a través del análisis de correlaciones probar la primera hipótesis. Para la prueba de las hipótesis dos y tres (las hipótesis se enuncian posteriormente) se propuso utilizar el subgrupo de las empresas mayores, cuyo comportamiento puede ser visto como un "modelo de crecimiento" para las microempresas. Este modelo también se empleó para realizar análisis factorial del tipo exploratorio y confirmatorio, identificando las principales variables del grupo de empresas mayores y agrupándolas en factores para establecer, a través de presuntas relaciones de causalidad entre ellos, la estructura del sistema y su diagrama de ruta para obtener una visualización gráfica y una mejor comprensión del fenómeno.

Este modelo parte del principio de que las empresas mayores han desarrollado a través de sus etapas de crecimiento mecanismos de éxito (o sobrevivencia) que 
marcan el camino que han seguido y que en un momento dado puede ser también seguido por las microempresas. Mediante dicho modelo se identificaron los factores que inciden en el desarrollo tecnológico y económico de las empresas mayores; también, por medio de la comparación de estos mismos factores en las microempresas, resultan los ajustes (alternativas de solución) que se requieren hacer en estos factores para estimular su desarrollo tecnológico y sus consecuentes efectos económicos.

Los sujetos de investigación son las empresas de los siete subsectores de los establecimientos de la ciudad de Chihuahua incluidos en el sector económico tres de la Clasificación Mexicana de Actividades y Productos (CMAP) del Instituto Nacional de Estadística, Geografía e Informática (2004), correspondiente a la industria manufacturera, y son las siguientes:

31.Productos alimenticios, bebidas y tabaco (alimentos).

32.Textiles, prendas de vestir e industria del cuero (textiles).

33. Industria de la madera y productos de madera, incluye muebles (madera).

34.Papel y productos de papel imprenta y editoriales (papel).

35.Sustancias químicas, productos derivados del petróleo y del carbón, de hule y de plástico (químicos).

36. Productos minerales no metálicos (minerales).

38. Productos metálicos, maquinaria y equipo, incluye instrumentos quirúrgicos y de precisión (metálicos).

Dichas empresas, en sus cuatro estratos de tamaños (micros, pequeñas, medianas y grandes), sumaban en la ciudad de Chihuahua alrededor de 650 empresas que emplean más de 70,000 trabajadores. El marco muestral se construyó con los listados del Sistema de Información Empresarial mexicano (SIEM), de asociaciones empresariales y del Centro de Información Económica y Social (CIES), publicados en el 2004. A continuación se presentan los principales datos para el cálculo de la muestra aleatoria estratificada por el tamaño de la empresa (Abad, 1993), considerando los valores de la variable de interés Nivel de tecnología del proceso (gtec_tot), para un error de un $8 \%$ de la media y un intervalo de confianza de un 95\% (Thompson, 1992); las fórmulas utilizadas fueron las siguientes:

$$
\mathrm{n}=\left(\sum \mathrm{N}_{\mathrm{i}}^{2} \cdot \mathrm{S}_{\mathrm{i}}^{2} / \mathrm{w}_{\mathrm{j}}\right) /\left(\sum \mathrm{N}_{\mathrm{i}} \cdot \mathrm{S}_{\mathrm{i}}^{2}+\mathrm{B}^{2} \cdot \mathrm{N}^{2} / \mathrm{Z}^{2}\right) \mathrm{y} \mathrm{n}_{\mathrm{i}}=\mathrm{n} \cdot \mathrm{w}_{\mathrm{i}}
$$


donde $\quad \mathrm{w}_{\mathrm{i}}=\mathrm{N}_{\mathrm{i}} \cdot \sigma_{\mathrm{i}} / \sum \mathrm{N}_{\mathrm{i}} \cdot \sigma_{\mathrm{i}} \quad$ (óptimo de Neyman), "n" es el tamaño de la muestra, "N" el de la población, "Si" la varianza de la variable de interés del estrato $i$, "w" la proporción del estrato, "B" el sesgo, "ni" la muestra del estrato $i$ y "Oi" la desviación estándar del estrato $i$.

\section{Cuadro 1}

\section{Determinación del tamaño de la muestra de las empresas manufactureras}

\begin{tabular}{|c|c|c|c|c|c|}
\hline Extrato & Poblaciow (N) & Meniso & $\begin{array}{l}\text { Desriacribe } \\
\text { endeshyr }\end{array}$ & $\begin{array}{l}\text { Ifueswa } \\
\text { de colveswo }\end{array}$ & $\begin{array}{c}\text { Musiona } \\
\text { nes' }\end{array}$ \\
\hline Misro & 295 & 22.1 & 13.2 & 44 & 59 \\
\hline Pequeda & 197 & 31 & 14.2 & 31 & 44 \\
\hline Mediana & 69 & 57.1 & 14.6 & 12 & 12 \\
\hline Grande & 63 & 99.6 & 14.9 & 10 & 10 \\
\hline Tecal & 624 & 32.4 & 20.2 & 96 & 125 \\
\hline
\end{tabular}

Se observa que la muestra real de 125 empresas (que superó con 29 empresas a la muestra calculada) representa aproximadamente un $20 \%$ del total de empresas de este sector industrial. En esta muestra se encuentran nueve empresas maquiladoras, de las cuales siete son grandes y dos medianas. En el cuadro 2 se presenta la distribución de las empresas de la muestra de acuerdo con sus subsectores o ramos de actividad.

Cuadro 2

Distribución de las empresas de la muestra por subsector y tamaño

\begin{tabular}{|c|c|c|c|c|c|c|c|c|}
\hline Torwato & Aînesenos & Muskers & Mefilices & Minnanas & Pagd & Puinion & Tesm & Totaf \\
\hline Micro & 2 & 6 & 18 & 10 & $\mathrm{~B}$ & 3 & 12 & 59 \\
\hline Pequeha & 5 & 10 & 7 & 1 & 7 & 6 & 8 & 44 \\
\hline Mediana & 1 & 1 & 3 & & 3 & 3 & 1 & 12 \\
\hline Grinde & & & 5 & & 1 & 3 & 1 & 10 \\
\hline Tetel & 8 & 17 & 33 & 11 & 19 & 15 & 22 & 125 \\
\hline
\end{tabular}

Fueme: Elaboracion del aisor

Las principales variables por medir y analizar son las directamente relacionadas con el problema y objetivos de investigación como son:

- La inversión anual en capacitación por empleado (Int_capa), que representa el monto total anual de la inversión en capacitación por empleado. Se calcula dividiendo el monto total anual invertido en capacitación dividido entre el número de trabajadores de la empresa (Craig, 1996). 
- La inversión en equipo administrativo (Inv_Adm), que representa el monto total de la inversión en equipamiento administrativo, que en su mayor parte es en tecnologías de información (TI); se calcula a precios de reposición.

- La inversión en equipo administrativo por trabajador (Invadptr), se calcula dividiendo la variable anterior (Inv_admi) entre el número de trabajadores.

- La inversión en equipo productivo (Inv_prod), que representa el monto total de la inversión en equipamiento del taller de producción, el cual se calcula a precios de reposición.

- Grado de automatización del proceso administrativo (gap_admi), que representa el nivel de automatización en los procesos del área administrativa de la empresa; se trata de un índice construido a partir de los reactivos del instrumento, el cual se expresa como un porcentaje del puntaje promedio de estos reactivos (Agresti, 1990).

- Grado de automatización del proceso productivo (gap_prod), que representa el nivel de automatización de las operaciones en el proceso productivo de la empresa; se trata de un índice construido a partir de los reactivos del instrumento, el cual se expresa como un porcentaje del puntaje promedio de estos reactivos.

- Nivel de empleo de las tecnologías organizativas (tec_blan), que representa la intensidad de utilización de las denominadas tecnologías blandas u organizativas, que se refieren a las nuevas formas de organizar el trabajo en general de la empresa como sistemas de calidad, el trabajo en equipos, etc.; se trata de un índice construido con base en los reactivos del instrumento, que se expresa como un porcentaje del puntaje promedio de ellos. Estas tecnologías "blandas" (traducción del inglés de soft technologies) están orientadas a los métodos o sistemas de organización del trabajo, por lo cual también son denominadas tecnologías sociales (Sanmartín, 1990).

- Nivel tecnológico del proceso de la empresa (gtec_tot), que representa el grado tecnológico total del proceso de la empresa; se trata de un índice construido con base en las tres variables anteriores correspondientes al grado de automatización de los procesos administrativo y productivo (tecnologías duras) y el nivel de empleo de las tecnologías organizativas (tecnologías blandas), el cual se expresa como un porcentaje del puntaje promedio de estas variables. Estas tecnologías difieren del concepto de "la innovación" y sólo son introducidas al mercado en forma indirecta a través de los productos (Corona, 1997).

- Valor agregado por empleado (Int_vag), que representa el valor agregado económico anual de la empresa por trabajador; se calcula sumando los 
montos anuales de la nómina y las utilidades y el resultado se divide por el número de trabajadores.

El instrumento consistió en un cuestionario formado básicamente por dos partes: la económica y la tecnológica; para esta última se construyeron unos índices (tecnologías en relación al área administrativa, al área productiva y las denominadas organizativas o blandas) con base en escalas del tipo Likert expresados en términos porcentuales, las cuales se describen a continuación.

a) Grado de automatización del proceso administrativo: se emplearon doce reactivos sobre la automatización (grado de empleo de la computadora) para la elaboración de la correspondencia, la contabilidad, la nómina, las órdenes a proveedores, el inventario, la facturación, el control de clientes, el tipo de servicio de conexión en red, el manejo de bases de datos, la existencia de una página de internet de la empresa y el empleo del comercio electrónico.

b) Grado de automatización del proceso productivo: se emplearon cinco reactivos sobre la automatización (grado de empleo de la computadora y máquinas) en el diseño de productos, los requerimientos de compra, la manufactura, las pruebas y el empaque.

c) Grado de empleo de las tecnologías organizativas: se emplearon ocho reactivos sobre el grado de intensidad en el trabajo con las técnicas de control o sistema de administración de la calidad, trabajo en equipos, mejora continua, justo a tiempo, manufactura esbelta, calidad total, cinco "S" y seis sigma.

Se parte de los siguientes supuestos, soportados en la experiencia del autor:

1. La automatización en los procesos administrativos, productivos y las tecnologías blandas u organizativas, refleja el grado de avance tecnológico en sus procesos.

2. La capacitación juega un papel de primera importancia en el desarrollo tecnológico de las empresas.

3. Todas las empresas poseen un nivel tecnológico en sus procesos por pequeño que éste sea.

4. El valor agregado económico por trabajador en las empresas refleja los resultados económicos de las empresas.

Como hipótesis de investigación se plantean las siguientes relacionadas con las empresas de la industria manufacturera: 
1. Hipótesis 1 (H1): A mayor número de trabajadores se tiene un mayor nivel tecnológico del proceso de producción en las empresas.

2. Hipótesis 2 (H2): A mayor nivel tecnológico en el proceso de producción se tienen mejores resultados económicos en las empresas mayores, medidos a través del valor agregado por trabajador.

3. Hipótesis 3 (H3): Si H2 es cierta, entonces es posible conocer cuál de los tres componentes en que se subdivide el nivel tecnológico del proceso es el que tiene una mayor influencia en los resultados económicos de las empresas mayores, medidos a través del valor agregado por trabajador.

En cuanto a la limitación del estudio, debido a que la muestra se tomará de la industria manufacturera de la ciudad de Chihuahua, el alcance de los resultados de la investigación para efectos de la inferencia estadística será esta población objetivo. Sin embargo, una vez desarrollado el instrumento de medición del nivel tecnológico del proceso se podrá, mediante ligeras adaptaciones, aplicarse en la industria manufacturera de otros lugares.

\section{Resultados}

A continuación se presentan los resultados de acuerdo con su nivel estadístico (descriptivo, correlacional, de regresión y factoriales), así como las soluciones propuestas.

\subsection{Análisis descriptivo}

En cuanto a la inversión anual en capacitación por trabajador (Int_capa) se encontró que los valores promedio son de $\$ 1,388$ para las micros y de $\$ 1,820$ para las mayores, la cual es superior en un $31 \%$. El valor del indicador de la inversión anual en capacitación como un porcentaje de la nómina para las empresas micro es aproximadamente de $1 \%$, mientras que para las empresas mayores es del orden de un 2\% (Ollivier, 2002); sin embargo, para las empresas de clase mundial oscila alrededor de un $5 \%$.

Por lo que respecta al grado de automatización del proceso administrativo (Gap_admi), se encontró 25.8\% para las micros y de 52.8\% para las mayores, lo cual arroja un rezago de un $27 \%$. Este resultado es congruente con la relación positiva entre el tamaño de la organización y el empleo de las TIC encontradas en las pequeñas empresas de Turquía (Acar et al., 2005). 
En relación con el grado de automatización del proceso productivo, se encontró de 21.8\% para las micros y de $36.1 \%$ para las mayores, lo cual arroja un rezago de un $14.3 \%$. Estos resultados son congruentes con los encontrados en Canadá por Dodgson en una encuesta realizada en 4,200 establecimientos manufactureros en el 2001, en la cual se destaca que las empresas grandes son líderes en la adopción de herramientas de automatización (Pedroza, 2005). La razón principal por la que se considera que es mayor el grado de automatización en los procesos administrativos que en los productivos es la accesibilidad a las computadoras personales y sus programas especializados en temas administrativos comunes ya estandarizados y aplicables a todas las empresas, lo cual no es el caso de los procesos productivos que requieren, generalmente, de maquinaria especializada para cada proceso, y más cara, así como personal más capacitado. Sin embargo, con el fin de reducir los tiempos de respuesta en la fabricación, se recomienda integrar los sistemas administrativos con los productivos (Stroeken, 2001).

En cuanto al grado de empleo de tecnologías organizativas, se encontró de 20.4\% para las micros y de $39.3 \%$ para las mayores, lo cual arroja un rezago de un $18.9 \%$. Es interesante observar la fuerte aplicación que hacen las empresas grandes de la tecnología organizativa, dada la conciencia que tienen de los grandes beneficios que les aportan al incremento de la productividad y a los costos relativamente reducidos que implican. Se estima que existe una clara tendencia al empleo intensivo de estas tecnologías en las medianas y grandes empresas.

A continuación se muestran en una gráfica de "cajas" (box plot) los resultados de la variable Nivel tecnológico del proceso (GTEC_TOT), que sintetiza a las tres variables tecnológicas anteriores, para los cuatro tamaños de empresas que aparecen en el eje horizontal. 


\section{Figura 1}

\section{Nivel tecnológico del proceso por tamaño de empresa}

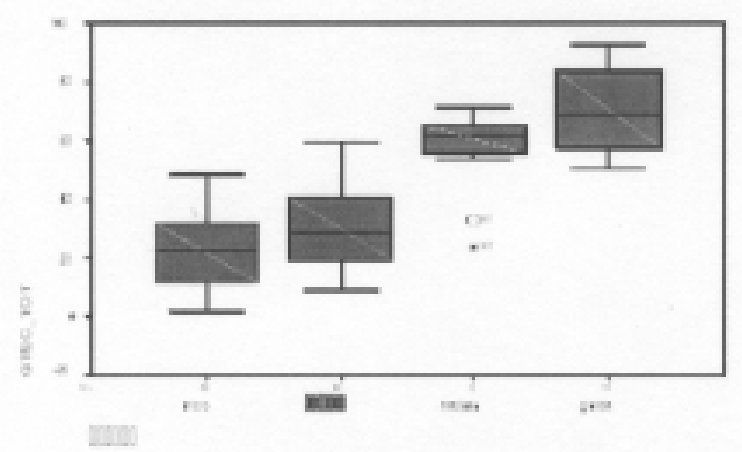

Se observa una relación directa entre el nivel tecnológico del proceso de la empresa y su tamaño. Esta variable, que representa la suma de las anteriores, muestra con claridad la fuerte desigualdad que existe en los niveles tecnológicos del proceso (en sus tres modalidades) entre las empresas micros, pequeñas, medianas y grandes. El valor de esta variable para las microempresas es de $22.1 \%$ y para las mayores de $41.9 \%$, lo cual arroja un rezago del orden de $19.8 \%$. Este resultado es congruente con los trabajos de la OCDE (1982), donde se reporta que debido a que la mayoría de las PYME (pequeña y mediana empresa) se enfrentan a obstáculos inherentes al uso y promoción de nuevas tecnologías, sólo una relativamente pequeña parte de ellas es capaz de iniciarse en avances científicos y de nuevas tecnologías.

\section{Cuadro 3}

Valores promedio de la variable Nivel tecnológico del proceso de la empresa por subsector y tamaño de empresa (El valor de la variable se expresa sobre base porcentual)

\begin{tabular}{|c|c|c|c|c|c|c|c|c|}
\hline Tounsho & Alenentos & Madera & Mrtalicos & Afinengirs & Pagn' & Qniwion & Textil & Total \\
\hline Micro & 34.26 & 13.64 & 27.63 & 1350 & 29.98 & 19.40 & 18.55 & 22.12 \\
\hline Pequefia & 28.87 & 28.49 & 32.99 & 13.02 & 44.60 & 27.94 & 26.48 & 31.02 \\
\hline Mediana & 65.59 & 70.97 & 66,90 & & 50.16 & 58.02 & 23.54 & 57,11 \\
\hline Grande & & & 72.73 & & 5382 & 76.29 & 50.17 & 69.65 \\
\hline Total & 3481 & 25.75 & 39.17 & & 39.81 & 41.92 & 23.10 & 32.42 \\
\hline
\end{tabular}

Focnte: Elabonacibn del wator 
Como se muestra en el cuadro 3, relativo al giro de las microempresas, los valores promedio mayores del nivel tecnológico lo presentan las empresas del subsector de químicos, papel y metálicos, mientras que los menores lo presentan las del subsector de minerales, textil y madera. En cuanto a su orientación al mercado interno o externo, se observa que los ramos o subsectores mayormente enfocados al mercado interno (González, 2002), como son los de minerales, textil y madera, tienen valores relativamente bajos en la tecnología del proceso.

Cabe señalar que a pesar del interés que puede tener distinguir en el análisis los giros de las empresas, hacerlo rebasaría el alcance del presente estudio, pues multiplicaría su complejidad; por ello, en lo subsecuente se continúa con el análisis considerando en bloque todos los subsectores, distinguiendo sólo el tamaño de las empresas, micros y mayores, dado el interés del estudio.

Referente al aspecto económico, la variable "valor agregado" (VA) es de gran importancia para este estudio, dado que se considera que refleja la salud y resultados económicos de las empresas al integrar esencialmente su nómina y sus utilidades. Se encontró que en términos generales este valor en relación con las ventas (valor agregado/ventas) es del orden de un 35\%.

Con el fin de poder comparar y evaluar esta variable, el tamaño se calcula en forma relativa, es decir, dividiendo el monto del valor agregado absoluto entre el número de trabajadores de la empresa para de esta forma poder considerarla como representante del resultado económico de la organización, independientemente de su tamaño. El principio en el que subyace lo anterior es considerar al valor agregado como un indicador que refleja el nivel tecnológico de la empresa, dado que una mayor tecnología implica por lo general mayores sueldos (mayor calificación del personal) y mayores utilidades. Congruente con lo anterior, se encontró que el valor promedio del valor agregado por trabajador es de $\$ 73,751$ para las micros y de $\$ 105,971$ para las empresas mayores, lo que representa una diferencia de $43.6 \%$.

\subsection{Análisis correlacional}

De acuerdo con lo expuesto en el apartado del método, a continuación se analiza únicamente el subgrupo de las empresas mayores. 


\section{Cuadro 4 \\ Correlaciones de la variable No de trabajadores y las variables tecnológicas}

\begin{tabular}{|c|c|c|c|}
\hline 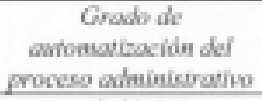 & $\begin{array}{c}\text { Grodb de } \\
\text { asrowatlacklow dd' } \\
\text { groceso prodatitho }\end{array}$ & $\begin{array}{c}\text { Gindo de emples of las } \\
\text { secenologias } \\
\text { onganizatives }\end{array}$ & $\begin{array}{c}\text { Grudo secwoligion do' } \\
\text { preceso }\end{array}$ \\
\hline $0.64^{* *}$ & $0.49 * 4$ & $0.59 *$ & $0.67 * 4$ \\
\hline
\end{tabular}

Fuente: Elaboracion del autor.

Noca: El nivel de significancia estadistica de us asteriseo " es p< 0.05 y dos "* p<0.01

La suma de las diferentes tecnologías representada por la tecnología del proceso tiene una correlación positiva de $0.67 * *$ con el tamaño de la empresa, lo cual es consistente con los resultados de la figura 1, confirmando que las empresas más grandes (con un mayor número de trabajadores) son también las que poseen mayores niveles de tecnología en sus procesos. Esta relación sugiere que, además de una relación de tipo causa-efecto lineal y en un sentido, se puede tener una relación circular en la que se retroalimentan mutua y positivamente el desarrollo tecnológico y el crecimiento de la empresa. En el cuadro anterior se observa una correlación positiva de 0.64 entre el grado de automatización del proceso administrativo y el tamaño de la empresa.

En cuanto a los resultados económicos, se encontraron correlaciones positivas entre el nivel tecnológico del proceso de las empresas mayores y el valor agregado (VA) de $0.64 * *$ y también con el valor agregado por trabajador de $0.6^{* *}$. Este resultado es congruente con la asociación positiva entre la innovación del producto o proceso y el desempeño del negocio reportada por David Smallbone et al. (2003) en pequeñas empresas inglesas e irlandesas. Estos resultados se pueden ver desde el enfoque de que la principal motivación que genera el cambio tecnológico en el proceso es el incremento de la productividad, la cual tiene una relación estrecha con el incremento del valor agregado, mediante la disminución de costos.

Sintetizando los puntos anteriores, debido a las correlaciones positivas entre tamaño de la empresa y nivel tecnológico por una parte y entre nivel tecnológico y el VA por trabajador por otra, se puede concluir que también hay una correlación positiva entre el tamaño de la empresa y el VA por trabajador, es decir, que el valor agregado por trabajador crece a medida que la empresa crece. 
El problema del rezago tecnológico de las microempresas

industriales y alternativas de solución

\subsection{Análisis de regresión}

Con el fin de identificar el peso o influencia que tienen las diferentes tecnologías en la determinación del valor agregado por trabajador, se realizó el siguiente análisis de regresión para las empresas mayores:

\section{Cuadro 5 \\ Análisis de regresión lineal entre el valor agregado por trabajador y las diferentes tecnologías en las empresas mayores}

\begin{tabular}{|c|c|c|c|c|c|c|}
\hline Dependiente & Predictorn & $\mathrm{R}^{2}$ & Coef. b & Coer. B & $F$ & $\sin$ \\
\hline \multirow[t]{4}{*}{$\begin{array}{l}\text { Valoe ayrepado } \\
\text { por trabajador }\end{array}$} & 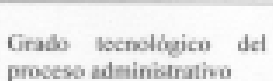 & 0.4 & 1,040 & 0.42 & 13.8 & 0.000 \\
\hline & $\begin{array}{l}\text { Grado wenologice del } \\
\text { proseso perdustino }\end{array}$ & & 386 & 0.19 & & 0.156 \\
\hline & 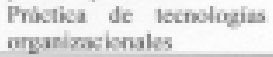 & & 279 & 0.12 & & 0.357 \\
\hline & Constante & & 24,631 & & & $0.6 \%$ \\
\hline
\end{tabular}

Se observa que la variable tecnológica con mayor influencia en la determinación del VA por trabajador es la del proceso administrativo, que corresponde al grado de automatización de las tareas administrativas, con un valor del coeficiente estandarizado beta (B) de 0.42 , seguida de las tecnologías en el proceso productivo en el taller y con menos influencia las tecnologías organizativas. Todas ellas con un coeficiente de determinación del VA por trabajador de un $40 \%\left(\mathrm{R}^{2}=0.4\right)$.

\subsection{Análisis factorial}

A continuación se presenta el análisis factorial (Kerlinger, 2002) para las empresas mayores, el cual consta de dos etapas: la exploratoria (EFA, Exploratory Factor Analysis del inglés) y la confirmatoria (CFA, Confirmatory Factor Analysis del inglés). Para la realización del análisis factorial exploratorio, a partir de la matriz de correlaciones entre las principales variables observadas, se identifican las que tienen una mayor relación para agruparlas por factores (variables de constructo, no observadas); posteriormente, de la relación presumible entre estos factores se pueden plantear relaciones causales que tienen que ser confirmadas (Byrne, 1994). 
En este caso, del análisis de correlaciones se identificaron las siguientes siete variables para las empresas mayores: Tamaño de la empresa (No_trab), Grado de automatización del proceso administrativo (Gap_adm), Inversión en capacitación por trabajador (Int_capa), Valor agregado por trabajador (Int_vag), Grado de automatización del proceso productivo (Gap_pro), Grado de empleo de las tecnologías organizativas (Tec_blan), Inversión en equipo administrativo por trabajador (Invadptr). Este análisis permitió identificar básicamente tres grupos o factores: el de las inversiones en equipo administrativo por trabajador (F3), el de las inversiones en capacitación por trabajador (F2) y otro de los resultados tanto en términos de los tres componentes tecnológicos como del valor agregado por trabajador (F1). Los factores identificados con sus cargas con la solución de Kaiser-Varimax y su comunalidad se muestran en el siguiente cuadro.

\section{Cuadro 6 \\ Factores, sus cargas y comunalidades de las principales variables de las empresas mayores}

\begin{tabular}{|c|c|c|c|c|}
\hline Variable & $\begin{array}{c}\text { Cargas del Facber I } \\
\left(\mathrm{F}_{1}\right)\end{array}$ & $\begin{array}{c}\text { Carges del Factor } 2 \\
\left(\mathrm{~F}_{1}\right)\end{array}$ & $\begin{array}{c}\text { Cargas del Facler } 3 \\
\left(F_{1}\right)\end{array}$ & Comunalindad \\
\hline No_trab & 0.72 & 0.13 & 0.15 & 0.56 \\
\hline Gap_adm & 0.78 & 0.15 & 0.14 & 0.65 \\
\hline Int_capa & 0.05 & 0.75 & 0.06 & 0.56 \\
\hline Int_vase & 0.67 & -0.02 & 0.18 & 0.48 \\
\hline Gap prod & 0.73 & -0.14 & 0.11 & 0.57 \\
\hline Tex_blan & 0.70 & 0.15 & 0.25 & 0.57 \\
\hline Ievadpir & 0.22 & 0.06 & a.2 & 0.57 \\
\hline
\end{tabular}

Fuente: Elaboración del aubs

El factor 1 tiene un valor "eigen" de 3.382 y una variación explicada de un 48.3\%; el factor 2, un valor "eigen" de 1.058 y una variación explicada de $15.1 \%$; y el factor 3, un valor "eigen" de 0.827 y una variación explicada de $11.8 \%$. Así, el total de los tres factores tiene una variación explicada de un 75.3\%. La lectura del cuadro anterior, de la relación entre factores a nivel exploratorio (que requerirá su confirmación posterior), sugiere que a mayores inversiones en la automatización del proceso administrativo por trabajador (F3) se requieren mayores inversiones en capacitación por trabajador (F2), lo que a su vez permite mayores niveles tecnológicos del proceso y un mayor valor agregado por trabajador (F1). 
El análisis factorial confirmatorio tiene como objetivo básico la confirmación o prueba de las relaciones planteadas en el análisis exploratorio anterior (Loehlin, 2004) para lo cual es necesario realizar un análisis de covarianza con el fin de identificar la relación entre los factores que determina la estructura del sistema. A continuación se muestran las ecuaciones de la solución estandarizada y su coeficiente de determinación $\mathrm{R}^{2}$ para las variables observadas y los factores, obtenidas con el paquete EQS 6.1., con el que se determinó el índice de ajuste (fittness) o bondad del modelo a los valores observados y que es de un 0.935 o sea de un 93.5\%. Este índice de ajuste normado, que es el de Bentler-Bonnet Normed Fit Index (NFI), se considera bueno cuando es superior a un 0.9 o sea un $90 \%$ (Bentler, 1995).

\section{Cuadro 7}

Ecuaciones de la solución estandarizada de la estructura de lasprincipales variables de las empresas mayores

\begin{tabular}{|c|c|c|c|c|}
\hline Variable & & & & $\mathrm{n}^{2}$ \\
\hline No_trab & & $=0.751\left(F_{1}\right)$ & $+0.66 \mathrm{E3}$ & 0.564 \\
\hline Gap adm & & $=0.844\left(F_{1}\right)$ & +0.536 E33 & 0.712 \\
\hline Int capa & & $-0.190\left(F_{2}\right)$ & $+0.052 \mathrm{E3} ?$ & 0.05 \\
\hline Ine_vang & & $=0.652\left(F_{1}\right)$ & $+0.749 \mathrm{E} 3 \mathrm{~B}$ & 0.430 \\
\hline Gap_prod & * & $=0.698\left(\mathrm{~F}_{i}\right)$ & $+0.716 \mathrm{E} 41$ & 0.487 \\
\hline Tec blan & & $=0.743\left\langle\mathrm{~F}_{1}\right)$ & $+0.650 \mathrm{FA2}$ & 0.552 \\
\hline Invadptr & & $=0.512\left(\mathrm{~F}_{3}\right)$ & +0.859 ES0 & 0.279 \\
\hline$F_{1}$ & & $=0.738\left\langle F_{2}\right\}$ & $+0.675 \mathrm{DI}$ & 0.544 \\
\hline$F_{2}$ & & $=0.060\left(\mathrm{r}_{2}\right)$ & +0.248122 & 0933 \\
\hline
\end{tabular}

Se observa que la variable observada que tiene una mayor influencia en esta estructura es el Grado de automatización del proceso administrativo (Gap_adm), con un coeficiente de determinación $\mathrm{R}^{2}$ relativamente alto de 0.712 , seguida por el tamaño de la empresa con coeficiente de 0.564 y por la práctica de las tecnologías organizativas (Tec_blan) de 0.552. A continuación se presenta el diagrama de ruta (del inglés Path Analysis), que muestra la estructura del sistema en un esquema gráfico, básicamente a través de la relación entre los factores, en donde F3 (independiente) es causa de F2 (dependiente) y éste a su vez es causa de F1 (dependiente). 


\section{Figura 2}

Diagrama de ruta de la estructura del modelo de las principales variables tecnológicas y económicas de las empresas mayores

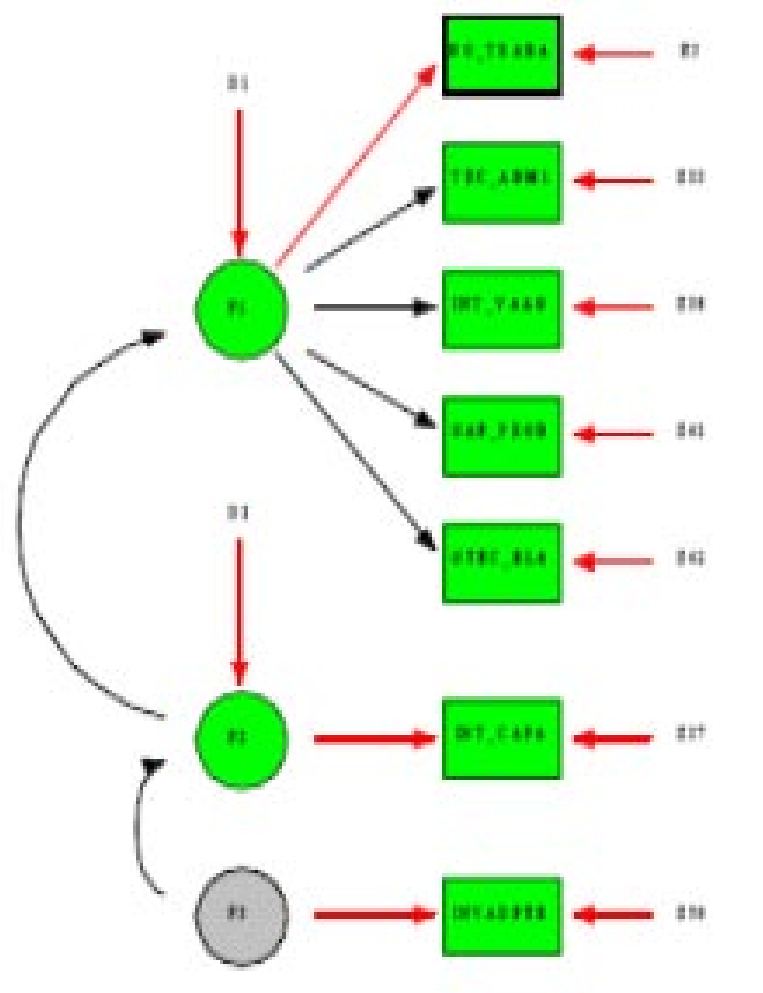

\subsection{Prueba de hipótesis}

Hipótesis 1 (H1): A mayor número de trabajadores se tiene un mayor nivel tecnológico del proceso de producción en las empresas. La hipótesis nula es: A mayor número de trabajadores no se tiene un mayor nivel tecnológico del proceso de producción en las empresas. Esta hipótesis se puede expresar estadísticamente a través de la correlación entre las variables Nivel tecnológico del proceso (a) y Número de trabajadores (b) cuando dicha correlación es menor o igual a cero $\left(\mathrm{r}_{\mathrm{ab}} \leq 0\right)$. Sin embargo, como se muestra en el cuadro 4, la correlación tiene un valor positivo de 0.67 (significancia $\mathrm{P}<0.01$ ); por lo tanto, la hipótesis nula se rechaza y la hipótesis de investigación se acepta. 
Hipótesis 2 (H2): A mayor nivel tecnológico en el proceso de producción se tienen mejores resultados económicos en las empresas, medidos a través del valor agregado por trabajador. La hipótesis nula es: A mayor nivel tecnológico en el proceso de producción no se tienen mejores resultados económicos en las empresas, medidos a través del valor agregado por trabajador. Esta hipótesis se puede expresar como sigue: la correlación entre las variables Nivel tecnológico del proceso (a) y valor agregado por trabajador (d) es menor o igual a cero, $\left(r_{a d} \leq 0\right)$. Sin embargo, como ya se mostró en el apartado del análisis correlacional 3.2, la correlación tiene un valor positivo de 0.6 (significancia $\mathrm{P}<0.01$ ); por lo tanto, la hipótesis nula se rechaza y la hipótesis de investigación se acepta.

Hipótesis 3 (H3): Si H2 es cierta, entonces es posible conocer cuál de los tres componentes en que se subdivide el nivel tecnológico del proceso es el que tiene una mayor influencia en los resultados económicos de las empresas, medidos a través del valor agregado por trabajador, donde cada beta (B1, B2, B3), es el coeficiente estandarizado de regresión correspondiente a cada una de las tres diferentes tecnologías. La hipótesis nula es: Si H2 es cierta, entonces no es posible conocer cuál de los tres componentes en que se subdivide el nivel tecnológico del proceso es el que tiene una mayor influencia en los resultados económicos de las empresas, medidos a través del valor agregado por trabajador $(\mathrm{B} 1=\mathrm{B} 2=\mathrm{B} 3)$. Tal como se muestra en el cuadro 5, los coeficientes estandarizados beta que determinan el peso o la influencia de cada una de las variables predictoras sobre la variable dependiente son de, $\mathrm{B} 1=0.42$ con una significancia $\mathrm{P}<0.01$ para el grado tecnológico del proceso administrativo, de B2 $=0.19$ para el grado tecnológico del proceso productivo y de $\mathrm{B} 3=0.12$ para la práctica de tecnologías organizacionales. Dado que los valores de beta son diferentes, $(\mathrm{B} 1 \neq \mathrm{B} 2 \neq \mathrm{B} 3)$, las distintas tecnologías tienen cada una un diferente peso o influencia sobre la variable dependiente, por lo que la hipótesis nula se rechaza y la hipótesis de investigación se acepta; el mayor valor de beta es el de la variable predictora de mayor influencia, que en este caso corresponde a B1 $(0.42$, con $\mathrm{P}<0.01)$, esto es, el grado tecnológico del proceso administrativo.

\subsection{Alternativas de solución}

Del análisis correlacional se observó que, en el caso de las empresas mayores, a medida que la empresa crece, también lo hacen su valor agregado por trabajador y sus niveles tecnológicos de los procesos en general, pero particularmente el 
administrativo. El análisis factorial permitió establecer una relación causal de los factores, que puede ser expresada como: Las inversiones en la automatización del proceso administrativo conducen a una mayor inversión en capacitación por trabajador, las cuales estimulan el crecimiento de la empresa y generan un mayor nivel tecnológico del proceso (administrativo, productivo y organizativo) y del valor agregado por trabajador.

Con base en lo anterior, las alternativas de solución que se proponen para disminuir el rezago tecnológico e incrementar el valor agregado de las microempresas manufactureras son en orden decreciente de importancia las siguientes:

1. El incremento significativo en la inversión en equipamiento para las tecnologías orientadas a la automatización del proceso administrativo. Como se vio, son estas tecnologías las que tienen un mayor impacto en la productividad de las empresas, la cual se ve traducida en el incremento del valor agregado por trabajador. Este resultado es congruente con las recomendaciones de poner énfasis en las tecnologías de procesos administrativos en las PYME (Bernal, 1999). En cuanto a las diferentes fases por las que debe pasar el proceso de adquisición y dominio de las tecnologías de información (del inglés IT), se han establecido los modelos de crecimiento de Nolan (1979), Venkatraman (1991) y más recientemente el de Tan (1995), el cual establece cinco niveles: integración funcional, integración entre funciones, integración del proceso, rediseño del proceso de negocios y la redefinición del alcance del negocio (Stroeken, 2001).

2. El incremento significativo en la capacitación, lo cual tiende a incrementar el capital humano de la empresa en general; pero en este caso particular tiene sentido desde el punto de vista de que las nuevas tecnologías en información y comunicaciones (TIC), entre las que se encuentran las relativas al proceso administrativo, requieren de la actualización constante del personal a través de la capacitación.

3. La incorporación intensiva de las denominadas tecnologías organizativas en los procesos. Ésta es igualmente una variable estrechamente relacionada con el crecimiento de la empresa, tal como lo muestra el análisis correlacional y es después de las tecnologías administrativas la que tiene una mayor influencia en el modelo de estructura. Por otra parte, en congruencia con el punto anterior, las tecnologías organizativas implican igualmente inversión en capacitación, debido a su naturaleza intangible que no requieren prácticamente inver- 
siones en equipamiento sino esencialmente en la formación de recursos humanos.

En cuanto a la forma de aplicar las medidas de estas propuestas de solución, tal como lo menciona Alejandro Mungaray (2002), lo preferible es hacerlo a través de las políticas industriales regionales, que dan más posibilidades de promoción económica a las micro y pequeñas empresas en torno a nichos de mercado que permitan obtener más valor agregado para acelerar los procesos de aprendizaje, inversión y crecimiento. Cabe mencionar, en términos generales, estas soluciones son congruentes con las recomendaciones sobre el tema que hacen los organismos internacionales, como la Organización para la Cooperación y el Desarrollo Económicos (OCDE, 1982 y 1996) y la Organización de las Naciones Unidas para el Desarrollo del Comercio (UNCTAD, 2003).

\section{Conclusiones}

En relación con el diagnóstico de la problemática, se constató que el rezago tecnológico en los procesos de las microempresas, en comparación con las mayores, es en general considerable en las tres áreas tecnológicas analizadas, particularmente en las del proceso administrativo. Por otra parte, se observó también que en las microempresas es relativamente baja la inversión en capacitación e igualmente lo es su resultado económico, medido a través del valor agregado.

En relación con las alternativas de solución de la problemática anterior, el análisis estadístico permitió constatar que a medida que la empresa crece también lo hace su valor agregado por trabajador y sus niveles tecnológicos de los procesos en general. Se identificó que la inversión que más influye para elevar la tecnología del proceso en general es la efectuada en el equipamiento de las tecnologías orientadas a la automatización de las tareas administrativas, que en forma genérica están incluidas en las tecnologías de información y comunicaciones (TIC). El análisis factorial y el modelo de estructura permitieron establecer que las inversiones en la automatización del proceso administrativo conducen a la inversión en capacitación por trabajador y éstas a su vez estimulan el crecimiento y generan un mayor nivel tecnológico del proceso y del valor agregado por trabajador. Con base en lo anterior, las alternativas de solución que se proponen para disminuir el rezago tecnológico e incrementar el valor agregado de las microempresas manufactureras son en orden (decreciente) de prioridad: 
1. El incremento significativo en la inversión en equipamiento para la automatización del proceso administrativo con el fin de disminuir el rezago en estas tecnologías que actualmente es del orden de un $27 \%$.

2. El incremento en la capacitación por trabajador; se observó que la inversión en capacitación es del orden del 1\% de la nómina cuando el valor de las empresas mayores es de un $2 \%$ y de las internacionales (World Class) del orden de un $5 \%$.

3. La incorporación intensiva de las denominadas tecnologías organizativas en los procesos, en las cuales igualmente las micro tienen un rezago significativo de un $19 \%$. Estas tecnologías se caracterizan por su alta rentabilidad por ser de bajo costo y altos beneficios potenciales en materia de productividad.

De acuerdo con el modelo de crecimiento propuesto en el presente estudio, estas medidas deberán de conducir hacia el crecimiento de las microempresas por medio de:

- Un incremento en el nivel tecnológico de los procesos en general, particularmente del administrativo, el cual es el que mayor influencia tiene en el nivel tecnológico del proceso en general.

- Un incremento en el valor agregado por trabajador, lo que implica mejores sueldos y mayores utilidades para la empresa, con lo cual se puede cerrar el círculo virtuoso del crecimiento de la empresa al incrementarse la capacidad de hacer inversiones con recursos propios.

Como conclusión general, se puede decir que la presente investigación propone, a través de sus hallazgos, un camino para transformar el círculo vicioso del rezago tecnológico de las microempresas manufactureras en un círculo virtuoso de crecimiento por la vía del desarrollo tecnológico.

Finalmente, se puede decir que este estudio permite una mejor comprensión del problema, al ofrecer una referencia sobre la relación que existe entre el proceso del desarrollo tecnológico y los resultados económicos en las empresas dentro de un sector industrial donde dicha relación puede determinar su sobrevivencia al incrementar su productividad y, en consecuencia, su competitividad. 
El problema del rezago tecnológico de las microempresas

industriales y alternativas de solución

\section{Recomendaciones}

El instrumento propuesto de medición del nivel tecnológico de los procesos ha demostrado en buena medida su confiabilidad al ser consistente consigo mismo en las más de 200 aplicaciones realizadas; sin embargo, será necesario, a fin de conservar el instrumento vigente, depurarlo y actualizarlo con las diferentes técnicas aplicables al subsector o industria involucrada debido, principalmente, a la propia naturaleza de la tecnología, la cual es dinámica y cambiante por antonomasia.

Igualmente, se recomienda una investigación para identificar la relación entre el nivel tecnológico incorporado al producto a través de la innovación (Jasso, 2003) y a su proceso de fabricación, lo que sería un complemento del presente trabajo.

Finalmente, se recomienda ampliar el alcance de la presente investigación realizándola en el nivel estatal, regional y nacional en la industria manufacturera, con el fin de proporcionar información objetiva y actualizada a los tomadores de decisión de estos niveles para el diseño de políticas industriales y económicas en materia de ciencia y tecnología.

\section{Referencias bibliográficas}

Abad, A. y L. Servín, Introducción al muestreo, Limusa Noriega, México, 1993.

Acar, E. et al., "Use of ICT by small and medium sized enterprises in building construction", Construction Management \& Economics, Vol. 23, No. 3, 2005, pp. 713-722.

Agresti, A., Categorical Data Analysis, Ed. John Wiley \& Sons, New York, 1990.

APEC, "SME action plan launched to help small business deal with globalization", boletín de prensa M2pressWire, No 16PU3076870241, Daegu, Corea del Sur, 2005.

Bentler, P., Structural Equations Program Manual, Multivariate Software Inc., Encino, California, 1995. 
Bernal, L., "Lineamientos de política tecnológica para la pequeña y mediana empresa", Plan de desarrollo económico y social 1998-2002, Colombia, 1999.

Berumen, S., Competitividad y desarrollo local, ESIC, Madrid, 2006.

Byrne, B., Structural Equation Modeling with EQS, SAGE Publications, Thousands Oaks, California, 1994.

Centro de Información Económica y Social, "Prontuario de indicadores socioeconómico Diciembre 2003", gobierno del estado de Chihuahua, 2004.

Consejo Nacional de Ciencia y Tecnología, Indicadores de actividades científicas y tecnológicas, Edición de bolsillo, México, 2004.

Corona, L., Cien empresas innovadoras en México, Miguel A. Porrúa, México, 1997.

Craig, L., The ASTD Training \& Development Handbook, 4ta ed., Mc Graw Hill, New York, 1996.

Gaynor, G., Handbook of technology management, Mc Graw-Hill, New York, 1996.

González, M., La industrialización en México, UNAM-IIE-Porrúa, México, 2002.

Hansen, O. et al., "Environmental Innovations in Small and medium Sized Enterprises", Technology Analysis \& Strategic Management, Carfax Publishing, Vol 14, No. 1, 2002, pp. 37-54.

Instituto Nacional de Estadística Geografía e Informática, Censo económico 2004, industria manufacturera, México, 2005.

- Estadísticas sobre ciencia y tecnología, www.inegi.gob.mx, México, 2005.

Jasso, J., "La trayectoria empresarial: reflexiones teóricas y metodológicas a partir del estudio de la innovación tecnológica", ponencia presentada en el VII Foro de Investigación, organizado por la Facultad de Contaduría y Administración, UNAM, México, 2003.

Kerlinger, F. y H. Lee, Investigación del comportamiento. Métodos de investi gación en ciencias sociales, 4a Ed, Mc Graw Hill, México, 2002.(A) 